\title{
Erratum to: Time-dependent decreases in nucleus accumbens AMPA/NMDA ratio and incubation of sucrose craving in adolescent and adult rats
}

Danielle S. Counotte • Christopher Schiefer • Yavin Shaham • Patricio O'Donnell

Published online: 29 November 2013

(C) Springer-Verlag Berlin Heidelberg 2013

Erratum to: Psychopharmacology

DOI 10.1007/s00213-013-3294-3

In the published paper of Counotte et al., there was an error in figure $3 \mathrm{H}$, obscuring the bars displaying the rectification index in adult rats. The authors apologize for this error and we reproduce the corrected figure 3 here.

The online version of the original article can be found at http:// dx.doi.org/10.1007/s00213-013-3294-3.

D. S. Counotte $(\varangle) \cdot$ C. Schiefer $\cdot$ P. O'Donnell

Department of Anatomy and Neurobiology, University of Maryland,

School of Medicine, 20 Penn St, Rm S-251, Baltimore, MD 21201,

USA

e-mail: d.s.counotte@gmail.com

Y. Shaham

Behavioral Neuroscience Branch, IRP-NIDA, Baltimore, MD, USA 
A water

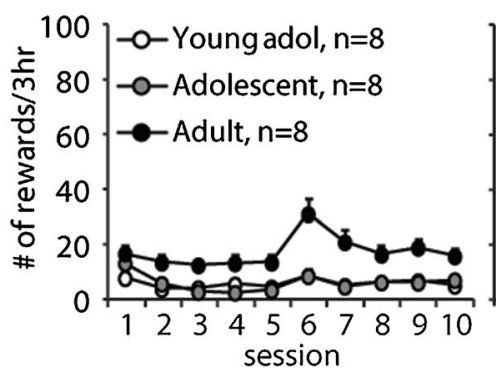

C 1-3 days abstinence

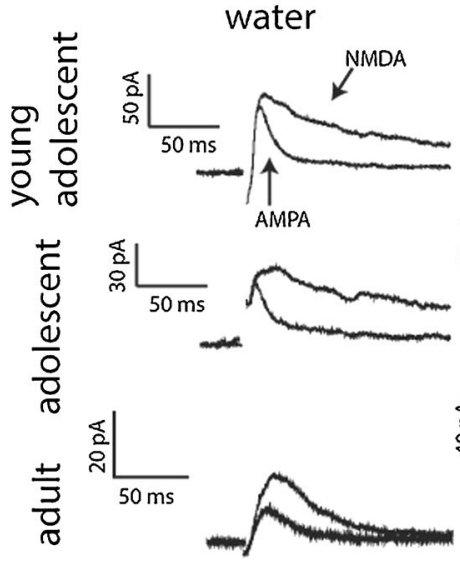

water

F 21 days abstinence

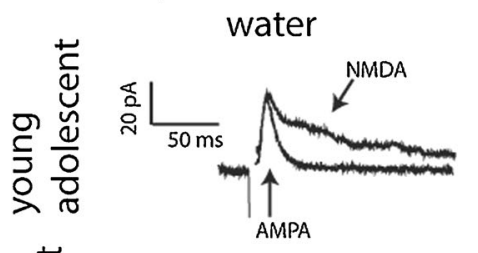

䓂
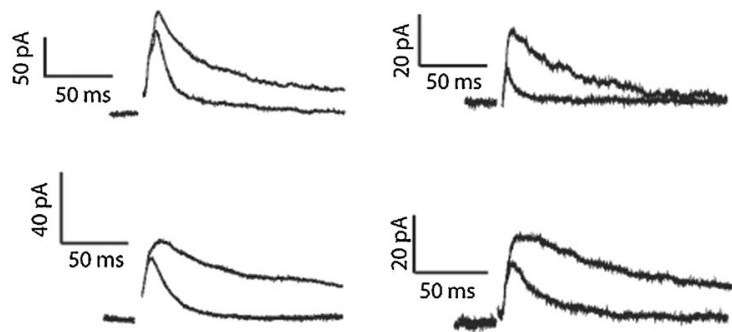

sucrose

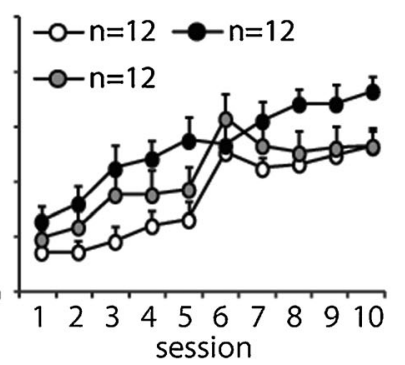

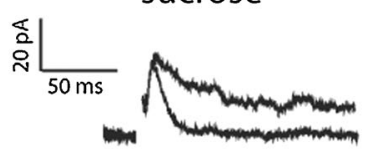

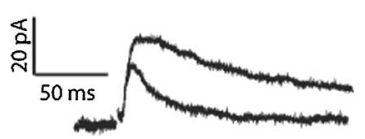

B

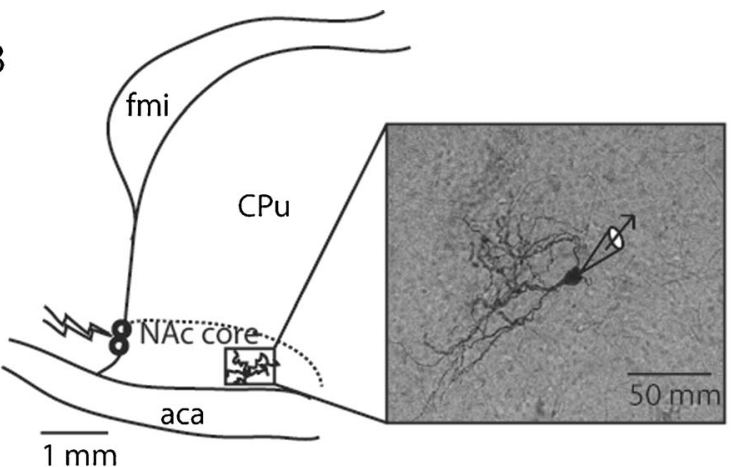

$E$

Fig. 3 AMPA/NMDA ratios in the nucleus accumbens core after 1-3 days or 3 weeks of abstinence from sucrose or water self-administration. (A) Mean \pm sem number of earned rewards in rats trained to self-administer water (left) or sucrose (right) during the training phase. Brains were taken after 1-3 days or 21 days of abstinence without testing for cueinduced reward seeking. (B) Schematic of a parasaggittal section through the nucleus accumbens core illustrating the relative placement of the stimulating electrode (bolt symbol) and recorded Neurobiotin-stained medium spiny neuron (inset) with respect to the forceps minor (fmi) and anterior commisure (aca). (C) Representative traces of medium spiny neurons held at $+40 \mathrm{mV}$ in the absence (both AMPA and NMDA) and presence (only AMPA) of the NMDAR antagonist AP5 in rats after 1-3 days of abstinence from self-administered water or sucrose at the three

G

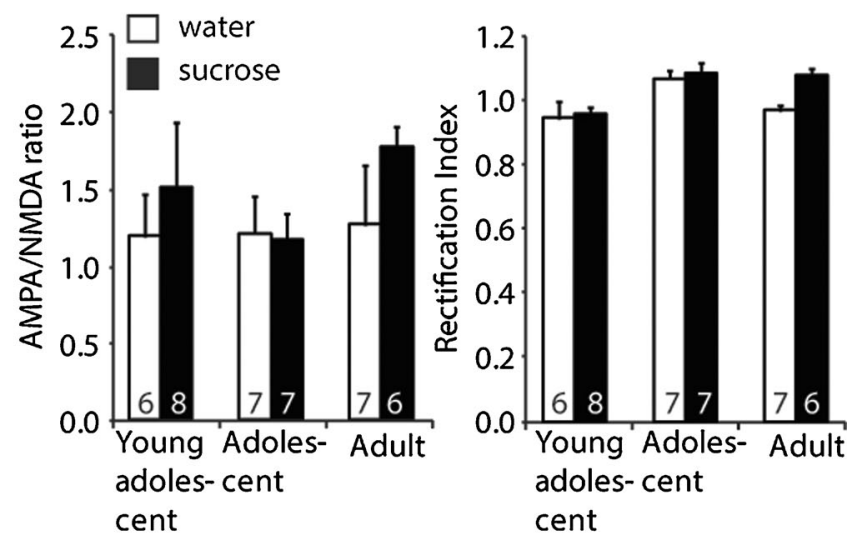

D

$\mathrm{H}$
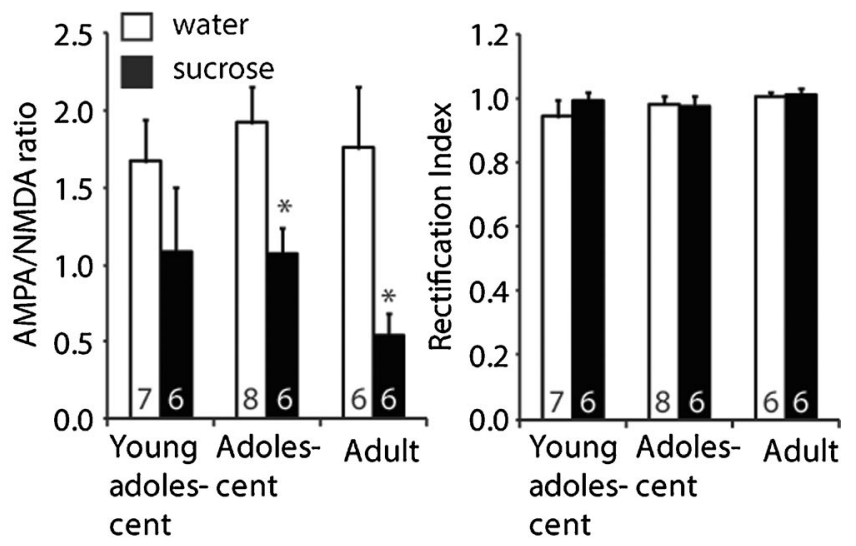

different age groups. Stimulation artifacts were removed for clarity. (D) Mean \pm sem AMPA/NMDA in NAc after 1-3 days of abstinence from selfadministered water (white bars) or sucrose (black bars). The numbers indicate cells per group ( $\mathrm{n}=4-6$ rats per group). No more than 2 cells per rat were used. (E) Rectification index after 1-3 abstinence days. (F) Representative traces of medium spiny neurons after 3 weeks of abstinence from water or sucrose self-administration in the three different age groups. (G) Mean \pm sem AMPA/NMDA ratio in NAc MSNs after 21 days of abstinence. The numbers indicate cells per group $(\mathrm{n}=\mathbf{4 - 6}$ rats per group). No more than 2 cells per rat were used. (H) Rectification index after 21 abstinence days. * Different from the Water condition within each age group, $\mathrm{p}<0.05$. 